Global Journal of Pure and Applied Mathematics.

ISSN 0973-1768 Volume 13, Number 9 (2017), pp. 6919-6928

(C) Research India Publications

https://dx.doi.org/10.37622/GJPAM/13.9.2017.6919-6928

\title{
A Mixed Asymmetric Laplace Moving Average Process
}

\author{
Bindu Krishnan \\ Research and Development Centre, \\ Bharathiar University, Coimbatore, Tamil Nadu, India. \\ Dais George \\ Catholicate College, Pathanamthitta, Kerala, India.
}

\begin{abstract}
In this paper, we define and study a first order moving average model with Laplace marginal distributions. Extensions to higher orders are discussed. A first order moving average process with mixed Laplace distributions as marginal is developed and studied. The model helps us to simulate mixed Laplace distribution with negative weights. We also introduce a first order moving average process as the mixture of asymmetric Laplace marginals.
\end{abstract}

AMS subject classification: 62M10, 62E99.

Keywords: Asymmetric Laplace distribution, Autoregressive process, Laplace distribution, Moving average process, Time series models.

\section{Introduction}

In modern field of research, the assumption of normality has played a vital role for the development of time series models. However, the need for non-Gaussian marginals has been long felt from the fact that many naturally occurring time series are nonGaussian.The Laplace distribution is an alternative for this, due to its application in modeling phenomena with "heavier than normal tails". The probability density function and characteristic function of standard Laplace random variable are respectively, given by

$$
f(x ; \sigma)=\frac{1}{2} e^{-|x|} ;-\infty<x<\infty
$$


and

$$
\phi_{X}(t)=\frac{1}{1+t^{2}}
$$

As the Laplace distribution is not appropriate for modeling data with asymmetric empirical distributions, skewed generalizations of Laplace distribution are considered for modeling by various researchers. Asymmetric Laplace distribution introduced by Kozubowski and Podgórski (2000) is a skewed version of symmetric Laplace distribution and a subclass of geometric stable distributions. They have stability properties and are convenient in applications, as their densities have explicit forms and the estimation procedures are easily implemented. This class of distributions is well suited for modeling phenomena where the variable of interest results from a large number of independent innovations, while the empirical distributions appears to be asymmetric, peaky and has tails heavier than those allowed by normal distribution. The probability density function of an $\operatorname{AL}(\mu, \sigma)$ distribution defined in Kotz et al. (2001) is

$$
f(x ; \mu, \sigma)=\frac{1}{\sigma} \frac{\kappa}{1+\kappa^{2}} \begin{cases}\exp \left[-\frac{\kappa}{\sigma} x\right], & x \geq 0 \\ \exp \left[\frac{1}{\kappa \sigma} x\right], & x<0\end{cases}
$$

where

$$
\kappa=\frac{2 \sigma}{\mu+\sqrt{4 \sigma^{2}+\mu^{2}}},-\infty<\mu<\infty, \sigma>0 .
$$

and the characteristic function is

$$
\phi_{X}(t)=\frac{1}{1+\sigma^{2} t^{2}-i \mu t} .
$$

Various forms of autoregressive models with non-Gaussian marginal distributions have been introduced by several authors in the past decades. But the moving average models with non-Gaussian marginal distributions have not investigated much in depth. Starting with the pioneering work of Gaver and Lewis (1980), non- Gaussian autoregressive models with different stationary marginal distribution are developed by various researchers. Andel (1983) and Dewald and Lewis (1985) developed and studied the autoregressive models for real valued variables using Laplace marginal distribution. Jayakumar et al. (1995) introduced autoregressive processes with $\alpha$ Laplace (Linnik) distribution as marginal distribution. Mathew and Jayakumar (2003) developed the autoregressive processes associated with generalized Linnik distribution. Seetha Lekshmi and Jose (2004) introduced autoregressive process with geometric Laplace marginal distribution and generalized to geometric $\alpha$ Laplace marginal. Kuttykrishnan and Jayakumar (2006) proposed an autoregressive model with bivariate semi $\alpha$ Laplace marginal. Jayakumar and Kuttykrishnan (2007) and Jayakumar et al.(2012) developed autoregressive models with asymmetric Laplace distribution as marginals and discussed various applications in modeling currency exchange rate, interest rate, stock price changes etc. 
Lawrance and Lewis (1977) introduced the first order moving average process in exponential variables known as EMA(1) model with structure

$$
X_{n}=\left\{\begin{array}{lll}
\beta \epsilon_{n-1} & \text { w.p } & \beta \\
\beta \epsilon_{n-1}+\epsilon_{n} & \text { w.p } & 1-\beta
\end{array} \quad 0 \leq \beta \leq 1 ; n=0, \pm 1, \pm 2, \ldots,\right.
$$

where $\left\{\epsilon_{n}\right\}$ is a sequence of independent and identically distributed (i.i.d) exponential random variables. This model has the first order autocorrelation

$$
\rho_{1}=\operatorname{Corr}\left(X_{n}, X_{n+1}\right)=\beta(1-\beta)
$$

and the higher order serial correlations are zero. They also defined a special type of linear model with random coefficients

$$
X_{n}=\beta \epsilon_{n}+I_{n} \epsilon_{n-1} ; 0 \leq \beta \leq 1,
$$

where $\left\{I_{n}\right\}$ is a sequence of i.i.d Bernoulli random variables taking values 1 and 0 with probabilities $1-\beta$ and $\beta$ respectively.

As an extension to the model (1.5), Jevremovic (1990) and Jevremovic (1991) developed the moving average model known as MAEX(1) which takes the form

$$
X_{n}=\left\{\begin{array}{lll}
\epsilon_{n} & \text { w.p } & p_{0} \\
\beta \epsilon_{n-1}+\epsilon_{n} & \text { w.p } & p_{1} \\
\beta \epsilon_{n-1} & \text { w.p } & p_{2},
\end{array}\right.
$$

where $0 \leq p_{0}, p_{1}, p_{2} \leq 1 ; p_{0}+p_{1}+p_{2}=1$ and $\left\{\epsilon_{n}\right\}$ 's are i.i.d random variables with exponential marginal distribution. Jevremovic and Malisic (1995) generalized the model (1.5) with the following structure

$$
X_{n}=\left\{\begin{array}{lll}
\epsilon_{n} & \text { w.p } & p_{0} \\
\beta_{1} \epsilon_{n-1}+\epsilon_{n} & \text { w.p } & p_{1} \\
\alpha_{1} \epsilon_{n-1} & \text { w.p } & q_{1} \\
\vdots & \vdots & \vdots \\
\beta_{t} \epsilon_{n-t}+\epsilon_{n} & \text { w.p } & p_{t} \\
\alpha_{t} \epsilon_{n-t} & \text { w.p } & q_{t}
\end{array}\right.
$$

where $\left\{\epsilon_{n}\right\}$ is a sequence of i.i.d random variables with exponential distribution while the parameters $\beta_{k}$ and $\alpha_{k}$ belong to the interval $(0,1)$ and $p_{0}+p_{1}+q_{1}+\cdots+p_{t}+q_{t}=1$. The purpose of this paper is to develop some linear time series models with Laplace and asymmetric Laplace marginal distributions. The paper is organized as follows. In Section 2, we developed and studied the first order Laplace moving average process with Laplace distribution as marginal distribution. Various extension to higher orders are also discussed in this section. In Section 3, we introduced and studied a first order moving average process with mixture of Laplace distributions as marginals and its extension to higher orders are obtained. A mixed asymmetric Laplace moving average model of order one is also derived in Section 4. Some applications of the distribution and the processes are discussed in Section 5. 


\section{Laplace Moving Average Process}

Let $\left\{X_{n}\right\}$ be a stationary sequence of random variables with first order moving average structure

$$
X_{n}=\left\{\begin{array}{lll}
\beta \epsilon_{n-1} & \text { w.p } & \beta^{2} \\
\beta \epsilon_{n-1}+\epsilon_{n} & \text { w.p } & 1-\beta^{2}
\end{array} 0 \leq \beta \leq 1 ; n=0, \pm 1, \pm 2, \ldots,\right.
$$

where $\left\{\epsilon_{n}\right\}$ is a sequence of i.i.d random variables.

In terms of characteristic function, the model (2.1) becomes

$$
\phi_{X_{n}}(t)=\beta^{2} \phi_{\epsilon_{n-1}}(\beta t)+\left(1-\beta^{2}\right) \phi_{\epsilon_{n-1}}(\beta t) \phi_{\epsilon_{n}}(t)
$$

If $\left\{\epsilon_{n}\right\}$ is a sequence of i.i.d standard Laplace random variables with characteristic function (1.2), then

$$
\begin{aligned}
\phi_{X_{n}}(t) & =\beta^{2} \phi_{\epsilon}(\beta t)+\left(1-\beta^{2}\right) \phi_{\epsilon}(\beta t) \phi_{\epsilon}(t) \\
& =\frac{1}{1+t^{2}} .
\end{aligned}
$$

which implies that $X_{n}$ has Laplace distribution. Hence, we can define the first order Laplace moving average LMA(1) process as

$$
X_{n}=\left\{\begin{array}{lll}
\beta L_{n-1} & \text { w.p } & \beta^{2} \\
\beta L_{n-1}+L_{n} & \text { w.p } & 1-\beta^{2}
\end{array} \quad 0 \leq \beta \leq 1 ; n=0, \pm 1, \pm 2, \ldots,\right.
$$

where $\left\{L_{n}\right\}$ is a sequence of i.i.d Laplace random variables.

The first order autocorrelation function of the model (2.2) is given by

$$
\rho_{1}=\beta\left(1-\beta^{2}\right)
$$

but the higher order serial correlations are zero and thus the spectral density of the process is

$$
f(\omega)=\frac{1}{\pi}\left[1+2 \beta\left(1-\beta^{2}\right) \cos \omega\right] ;-\pi \leq \omega \leq \pi .
$$

Also, we have $P\left[X_{n} \geq X_{n-1}\right]=P\left[X_{n} \leq X_{n-1}\right]=\frac{1}{2}$.

The joint characteristic function of $X_{n}$ and $X_{n+1}$ is given by

$$
\phi_{X_{n}, X_{n+1}}\left(t_{1}, t_{2}\right)=\frac{1+\beta^{2}\left(t_{1}^{2}+t_{2}^{2}+2 \beta t_{1} t_{2}\right)}{\left[1+\beta^{2} t_{1}^{2}\right]\left[1+t_{2}^{2}\right]\left[1+t_{1}^{2}+\beta^{2} t_{2}^{2}+2 \beta t_{1} t_{2}\right]},
$$

which implies $\phi_{X_{n}, X_{n+1}}\left(t_{1}, t_{2}\right) \neq \phi_{X_{n}, X_{n+1}}\left(t_{2}, t_{1}\right)$. Hence the process is not time reversible. 


\subsection{Extensions}

Let $\left\{L_{n}\right\}$ be a sequence of i.i.d Laplace random variables, then the second order Laplace moving average process $\operatorname{LMA}(2)$ is defined as

$$
X_{n}=\left\{\begin{array}{lll}
\beta_{2} L_{n} & \text { w.p } & \beta_{2}^{2} \\
\beta_{2} L_{n}+\beta_{1} L_{n-1} & \text { w.p } & \left(1-\beta_{2}^{2}\right) \beta_{1}^{2} \\
\beta_{2} L_{n}+\beta_{1} L_{n-1}+L_{n-2} & \text { w.p } & \left(1-\beta_{2}^{2}\right)\left(1-\beta_{1}^{2}\right),
\end{array}\right.
$$

where $0 \leq \beta_{1}, \beta_{2} \leq 1$ and $n=0, \pm 1, \pm 2, \ldots$.

The first and second order autocorrelations are

$$
\rho_{1}=\beta_{1}\left(1-\beta_{2}^{2}\right)\left[\left(1-\beta_{1}^{2}\right)\left(1-\beta_{2}^{2}\right)+\beta_{2}\right]
$$

and

$$
\rho_{2}=\left(1-\beta_{1}^{2}\right)\left(1-\beta_{2}^{2}\right) \beta_{2}
$$

respectively.

The higher order serial correlations of LMA(2) are zeroes. The model (2.6) can be generalized to Laplace moving average process of order $q$ with structure

$$
X_{n}=\left\{\begin{array}{lll}
\beta_{q} L_{n} & \text { w.p } & b_{q+1} \\
\beta_{q} L_{n}+\beta_{q-1} L_{n-1} & \text { w.p } & b_{q} \\
\vdots & \vdots & \vdots \\
\beta_{q} L_{n}+\beta_{q-1} L_{n-1}+\cdots+\beta_{1} L_{n-q+1} & \text { w.p } & b_{2} \\
\beta_{q} L_{n}+\beta_{q-1} L_{n-1}+\cdots+\beta_{1} L_{n-q+1}+L_{n-q} & \text { w.p } & b_{1},
\end{array}\right.
$$

where $0 \leq \beta_{1}, \beta_{2}, \ldots \beta_{q} \leq 1 ; n=0, \pm 1, \mp 2, \ldots$;

$$
b_{n}=\left\{\begin{array}{ll}
\beta_{q}^{2} & \text { for } \quad n=q+1 \\
\left(1-\beta_{q}^{2} \cdots\left(1-\beta_{n}^{2}\right) \beta_{n-1}^{2}\right. & \text { for } \quad q \geq n \geq 2 \\
\left(1-\beta_{q}^{2}\right) \cdots\left(1-\beta_{1}^{2}\right) & \text { for } \quad n=1
\end{array} \quad(q \geq 2)\right.
$$

$0 \leq b_{1}, b_{2}, \ldots, b_{q+1} \leq 1 ; b_{1}+b_{2}+\cdots+b_{q+1}=1$. and $\left\{L_{n}\right\}$ is a sequence of i.i.d Laplace random variables.

The autocorrelations for the model (2.9) clearly have the cut-off property associated with the moving average schemes. On routine computations, we derived the $h^{\text {th }}$ order autocorrelation of LMA(q) as

$$
\rho_{h}=\left\{\begin{array}{lll}
\sum_{j=1}^{q-h+1} \frac{b_{j}}{\beta_{j-1}} \frac{b_{j+h}}{\beta_{j+h-1}} & \text { for } & 1 \leq h \leq q \\
0 & \text { for } & q+1 \leq h<\infty .
\end{array}\right.
$$




\section{Mixed Laplace Moving Average Process}

Let $\left\{\epsilon_{n}\right\}$ be a sequence of i.i.d Laplace random variables. Then from the model (1.7), in terms of characteristic function, we have

$$
\begin{gathered}
\phi_{X_{n}}(t)=p_{0} \phi_{\epsilon_{n}}(t)+p_{1} \phi_{\epsilon_{n-1}}(\beta t) \phi_{\epsilon_{n}}(t)+p_{2} \phi_{\epsilon_{n-1}}(\beta t) \\
=p_{0} \frac{1}{1+t^{2}}+p_{1} \frac{1}{1+\beta^{2} t^{2}} \frac{1}{1+t^{2}}+p_{2} \frac{1}{1+\beta^{2} t^{2}} .
\end{gathered}
$$

Simplifying, we get,

$$
\phi_{X_{n}}(t)=\frac{\gamma}{1+t^{2}}+\frac{1-\gamma}{1+\beta^{2} t^{2}}
$$

where

$$
\gamma=\frac{1-\beta^{2} p_{0}-p_{2}}{1-\beta^{2}}
$$

Hence, $\left\{X_{n}\right\}$ is a sequence of mixture of Laplace random variables such that

$$
X_{n}=\left\{\begin{array}{lll}
L_{n} & \text { w.p } & \gamma \\
\beta L_{n} & \text { w.p } & 1-\gamma
\end{array} \quad 0 \leq \beta \leq 1 ; n=0, \pm 1, \pm 2, \ldots,\right.
$$

where $\left\{L_{n}\right\}$ is a sequence of Laplace random variables with characteristic function $\phi_{L_{n}}(t)=\frac{1}{1+t^{2}}$.

If $\beta^{2}>\frac{1-p_{2}}{p_{0}}$, then $\gamma$ will be negative or if $\beta^{2}>\frac{p_{2}}{1-p_{0}}$, then $1-\gamma$ will be negative and hence the distribution of $\left\{X_{n}\right\}$ is a mixture of Laplace random variables with negative weights. Hence using the model (1.7), it is possible to generate sequence of random variables that are mixtures of Laplace random variables with negative weights.

Based on (1.7) and (3.2), we define a first order mixed Laplace moving process as

$$
X_{n}=\left\{\begin{array}{lll}
\epsilon_{n} & \text { w.p } & p_{0} \\
\beta \epsilon_{n-1}+\epsilon_{n} & \text { w.p } & p_{1} \\
\beta \epsilon_{n-1} & \text { w.p } & p_{2}
\end{array}\right.
$$

where $0 \leq p_{0}, p_{1}, p_{2} \leq 1 ; p_{0}+p_{1}+p_{2}=1 ; 0 \leq \beta \leq 1$ and $\left\{\epsilon_{n}\right\}$ is a sequence of i.i.d Laplace random variables. 


\subsection{Extensions}

Now we consider the second order moving average model, which is a generalization of model (1.7) and has the form

$$
X_{n}=\left\{\begin{array}{lll}
\epsilon_{n} & \text { w.p } & p_{0} \\
\beta \epsilon_{n-1}+\epsilon_{n} & \text { w.p } & p_{1} \\
\beta \epsilon_{n-1} & \text { w.p } & p_{2} \\
\beta \epsilon_{n-2}+\epsilon_{n} & \text { w.p } & p_{3} \\
\beta \epsilon_{n-2} & \text { w.p } & p_{4}
\end{array}\right.
$$

where $0 \leq \beta \leq 1 ; 0 \leq p_{0}, p_{1}, p_{2}, p_{3}, p_{4} \leq 1 ; p_{0}+p_{1}+p_{2}+p_{3}+p_{4}=1$.

In the above model (3.4), if $\left\{\epsilon_{n}\right\}$ is an i.i.d sequence of Laplace random variables, then we get

$$
\phi_{X_{n}}(t)=\frac{\theta}{1+t^{2}}+\frac{1-\theta}{1+\beta^{2} t^{2}}
$$

where

$$
\theta=\frac{1-\beta^{2} p_{0}-p_{2}-p_{4}}{1-\beta^{2}}
$$

Hence, $\left\{X_{n}\right\}$ is a sequence of mixture of Laplace random variables.

If $\beta^{2}>\frac{1-p_{2}-p_{4}}{p_{0}}$, then $\gamma$ will be negative or if $\beta^{2}>\frac{p_{2}+p_{4}}{1-p_{0}}$, then $1-\gamma$ will be negative and hence the distribution of $\left\{X_{n}\right\}$ is a convex mixture of Laplace random variables with negative weights.

\section{Mixed Asymmetric Laplace Moving Average Process}

Let $\left\{X_{n}\right\}$ be a sequence of random variables with structure

$$
X_{n}=\left\{\begin{array}{lll}
\epsilon_{n} & \text { w.p } & p_{0} \\
\beta \epsilon_{n-1}+\epsilon_{n} & \text { w.p } & p_{1}
\end{array}\right.
$$

where $0 \leq \beta \leq 1 ; 0 \leq p_{0}, p_{1} \leq 1$ and $p_{0}+p_{1}=1$.

Then, using characteristic function,

$$
\phi_{X_{n}}(t)=p_{0} \phi_{\epsilon_{n}}(t)+p_{1} \phi_{\epsilon_{n-1}}(\beta t) \phi_{\epsilon_{n}}(t)
$$

. If $\left\{\epsilon_{n}\right\}$ is an i.i.d sequence of asymmetric Laplace random variables, then we have

$$
\begin{gathered}
\phi_{X_{n}}(t)=p_{0} \frac{1}{1+\sigma^{2} t^{2}-i \mu t}+p_{1} \frac{1}{1+\beta^{2} \sigma^{2} t^{2}-i \mu \sigma t} \frac{1}{1+\sigma^{2} t^{2}-i \mu t} \\
=p_{0} \frac{1}{1+i t \sigma \kappa} \frac{1}{1-i t \frac{\sigma}{\kappa}}+p_{1} \frac{1}{1+i t \beta \sigma \kappa} \frac{1}{1-i t \beta \frac{\sigma}{\kappa}} \frac{1}{1+i t \sigma \kappa} \frac{1}{1-i t \frac{\sigma}{\kappa}}
\end{gathered}
$$




$$
=\frac{\gamma_{1}}{1+i t \sigma \kappa}+\frac{\gamma_{2}}{1-i t \frac{\sigma}{\kappa}}+\frac{\gamma_{3}}{1+i t \beta \sigma \kappa}+\frac{\gamma_{4}}{1-i t \beta \frac{\sigma}{\kappa}}
$$

where

$$
\begin{array}{ll}
\gamma_{1}=\frac{p_{0} \beta \kappa^{2}\left(1-\beta-\kappa^{2}\right)+\kappa^{4}}{(1-\beta)\left(1+\kappa^{2}\right)\left(\beta+\kappa^{2}\right)} ; & \gamma_{2}=\frac{p_{0} \beta\left(\kappa^{2}-1-\beta \kappa^{2}\right)+1}{(1-\beta)\left(1+\kappa^{2}\right)\left(1+\beta \kappa^{2}\right)} ; \\
\gamma_{3}=\frac{-\beta^{2} \kappa^{4}\left(1-p_{0}\right)}{(1-\beta)\left(1+\kappa^{2}\right)\left(1+\beta \kappa^{2}\right)} ; & \gamma_{4}=\frac{-\beta^{2}\left(1-p_{0}\right)}{(1-\beta)\left(1+\kappa^{2}\right)\left(\beta+\kappa^{2}\right)}
\end{array}
$$

and

$$
\gamma_{1}+\gamma_{2}+\gamma_{3}+\gamma_{4}=1
$$

Hence, $\left\{X_{n}\right\}$ is a sequence of mixture of exponential random variables such that

$$
X_{n}=\left\{\begin{array}{lll}
-\sigma \kappa E_{1} & \text { w.p } & \gamma_{1} \\
\frac{\sigma}{\kappa} E_{2} & \text { w.p } & \gamma_{2} \\
-\beta \sigma \kappa E_{3} & \text { w.p } & \gamma_{3} \\
\frac{\beta \sigma}{\kappa} E_{4} & \text { w.p } & \gamma_{4}
\end{array}\right.
$$

where $\left\{E_{n}\right\}_{n=1,2,3,4}$ is a sequence of exponential random variables with characteristic function $\frac{1}{1-i t}$.

As Laplace distribution arises as the distribution of difference of two i.i.d exponential random variables, the structure (4.2) reduces to

$$
X_{n}=\left\{\begin{array}{lll}
A L_{n} & \text { w.p } & \delta \\
\beta A L_{n} & \text { w.p } & 1-\delta
\end{array}\right.
$$

where $0<\delta<1$ and $\left\{A L_{n}\right\}$ is an i.i.d sequence of asymmetric Laplace random variables. Hence, $\left\{X_{n}\right\}$ is a sequence of mixture of asymmetric Laplace random variables.

\section{Applications}

Laplace distribution have proved in literature, as a good alternative for normal distribution with a wide range of applications in time series modeling, financial modeling, communication engineering, image source modeling, gene expression data modeling etc. Asymmetric Laplace distribution also has got considerable attention among the researchers working in financial modeling, engineering sciences, share market return models, stochastic variance models and time series modeling. Damsleth and El-Shaarawi (1989) used first order autoregressive model with Laplace innovations to fit the data on sulphate concentration in the Turkey Lakes Watershed in Canada. Raillard et al. (2015) proposed a method to model time series that exhibit both horizontal and vertical asymmetries where the model is based on Laplace moving average which involves 
four parameters for the marginal distribution and a kernel. They also checked Laplace moving average model for fitting on a time series of sea waves. As the Laplace and asymmetric Laplace distributions are considered as best choice for modeling whenever data exhibiting heavier tails than Gaussian tails, the moving average processes discussed in this paper could be the good models for various time series data fitting. Also, the mixed Laplace moving average model can be used to simulate mixed Laplace distribution with negative weights.

\section{References}

[1] Andel J, (1983), Marginal distributions of autoregressive processes. Transactions of $9^{\text {th }}$ Prague Conference Information Theory, Academia, Praha, 127-135.

[2] Damsleth E and EI-Shaarawi AH, (1989), ARMA models with double exponentially distributed noise. Journal of Royal Statistical Society, B 51, 61-69.

[3] Dewald LS and Lewis PAW, (1985), A new Laplace second order autoregressive time series model NLAR(2). IEEE Transactions on Information Theory, 31, 645651.

[4] Gaver DP and Lewis PAW, (1980), First order autoregressive Gamma sequences and point processes. Advances in Applied Probability, 12, 727-745.

[5] Jayakumar K, Kalyanaraman K and Pillai RN, (1995), $\alpha$ Laplace processes. Mathematical and Computer Modeling, 22, 109-116.

[6] Jayakumar K and Kuttykrishnan AP, (2007), A time series model using asymmetric Laplace distribution. Statistics and Probability Letters, 77, 1636-1640.

[7] Jayakumar K, Kuttykrishnan AP and Kozubowski TJ, (2012) A first order autoregressive asymmetric Laplace process. Journal of Probability and Statistical Science, 10(1), 1-14.

[8] Jevremovic V, (1990), Two examples of non-linear processes with a mixed exponential marginal distribution. Statistics and Probability Letters, 10, 221-224.

[9] Jevremovic V, (1991), A note on mixed exponential distribution with negative weights. Statistics and Probability Letters, 11, 259-265.

[10] Jevremovic V and Malisic, (1995), On some moving average processes with exponentially distributed innovations. Journal of Italian statistical Society, 3, 337-343.

[11] Kotz S, Kozubowski TJ and Podgórski K, (2001), The Laplace distributions and Generalizations: A Revisit with Applications to Communications, Economics, Engineering and Finance. Birkhauser, Boston.

[12] Kozubowski TJ and Podgórski K, (2000), Asymmetric Laplace distribution. Math. Sci, 25, 37-46.

[13] Kuttykrishnan AP and Jayakumar K, (2006), Bivariate semi $\alpha$ Laplace distribution and processes. Statistical Papers, 49, 303-313. 
[14] Lawrance AJ and Lewis PAW, (1977), An exponential moving average sequence and point process(EMA(1)). Journal of Applied Probability, 14, 98-113.

[15] Nicholas Raillard, Marc Prevosto and Pierre Ailliot, (2015), Modeling Process Asymmetries with Laplace Moving Average, Computational Statistics and Data Analysis, 81, 24-37.

[16] Seetha Lekshmi V and Jose KK, (2004), An autoregressive process with geometric $\alpha$-Laplace marginals. Statistical Papers, 45, 337-350.

[17] Thomas Mathew and Jayakumar K, (2003), Generalized Linnik distributions and processes. Stochastic Modeling and Applications, 6, 27-37. 